\title{
Meta-Analysis of the Bacterial Detection Rate in Perianal Abscess
}

\author{
Peizheng Han1, Jingtao Sha2*, Shenghua Du', Xiangyan Yang1 \\ ${ }^{1}$ Shaanxi University of Chinese Medicine, Xianyang 712046, Shaanxi Province, China \\ ${ }^{2}$ Xi'an Hospital of Traditional Chinese Medicine, Xi'an 710021, Shaanxi Province, China \\ *Corresponding author: Jingtao Sha, 1245328255@qq.com
}

\begin{abstract}
Literatures on the distribution of bacteria in perianal abscess from different regions and at different times were systematically analyzed, and the distribution of pathogens in perianal abscess was meta-analyzed using STATA 12.0 statistical software. The results showed that the detection rate of Escherichia coli was 0.64 (95\% CI, 0.54-0.74), Klebsiella pneumoniae was 0.13 (95\% CI, 0.12-0.15), and Staphylococcus was 0.07 (95\% CI, 0.04-0.10).
\end{abstract}

Keywords: Perianal abscess; Meta-analysis; Bacteria; Flora; Escherichia coli; Klebsiella pneumoniae; Staphylococcus; Antibiotic

Publication date: September 2021; Online publication: September 30, 2021

\section{Introduction}

Perianal abscess is the abbreviation of perianorectal abscess. It is characterized by an acute and rapid onset. In physiological anatomy, it is an acute suppurative infection dominated by the space around the anal canal and rectum. Pain, swelling, and fever are considered to be the clinical manifestations of perianal abscess. As early as 1961, A.G. Parks, the Consultant Surgeon of London Hospital, introduced the hidden gland theory. Infection penetrates the anal wall through cracks or other wounds. Once the trace of infection is established, it enters the internal orifice through feces for maintenance. Intestinal microorganisms entering the anal canal will cause acute inflammation and spread along the internal longitudinal path (such as perianal abscess) ${ }^{[1]}$.

The study of the distribution of bacteria in perianal abscess is conducive to the clinical diagnosis, pathogenesis, and treatment of perianal abscess. Therefore, this study uses meta-analysis to combine the research results at home and abroad in order to provide a basis to guide the diagnosis and treatment of perianal abscess related diseases.

\section{Materials and methods}

\subsection{General information}

\subsubsection{Literature retrieval and retrieval strategy}

By searching 7 major databases at home and abroad, the included literatures were extracted after data screening, and literatures on the distribution of perianal abscess published in each database since 2010 were collected. The search criteria included English terms, such as "perianal," "perianorectal," "microbiology," and “bacteria,” as well as Chinese keywords, such as “肛周脓肿,” “肛管直肠周围脓肿,” “细菌,” and “菌 群.” 


\subsubsection{Inclusion criteria}

The inclusion criteria were as follows: (1) bacterial samples of pus from patients with a clear diagnosis of perianal abscess; (2) cross-sectional study as the research method; (3) clear number and type of bacterial culture as well as perfect original data; (4) literatures that were published after 2010.

\subsubsection{Exclusion criteria}

The exclusion criteria were as follows: (1) the number of specimens in the study was less than 70; (2) conferences, abstracts, and other literatures with incomplete pathogen data and were unable to conduct data analysis; (3) the publication time of the literature does not meet the time limit (including literatures that were published after 2010).

\subsection{Methods}

\subsubsection{Data extraction}

The basic information (author's name, publication time, country, region, sample size, bacterial classification, and sample number) of each document was extracted (Table 1). A total of 1,771 bacterial culture specimens of perianal abscess from 11 literatures were included in this study (30 invalid samples) ${ }^{[2-12]}$. The types of bacteria obtained in various literatures were different. Through screening and excluding the bacteria without research significance (number of bacteria detected was less than 3; bacterial culture of individual studies), it was concluded that the main pathogenic bacteria detected in various studies were Escherichia coli, Klebsiella pneumoniae, and Staphylococcus. The detection rates for these three pathogens were analyzed by meta-analysis.

Table 1. Basic information of each literature and flora distribution

\begin{tabular}{|c|c|c|c|c|c|c|c|c|c|c|c|c|c|}
\hline name & Publshing Time & Nation & sample size & male & femlae & years & Escherichia coli & Klebsiclla pacumoniase & Staphylococcus & streptococcus & Pseudomonas copper & enterococcus & Morganella \\
\hline Qian Cao & 2019 & China & 78 & 78 & 22 & $1-82$ & 45 & 11 & 8 & 0 & 0 & 2 & 0 \\
\hline Xinying Zhang & 2019 & China & 217 & - & - & - & 152 & 31 & 5 & 8 & 2 & 1 & 0 \\
\hline Feng Wu & 2018 & China & 280 & 250 & 30 & $4-80$ & 184 & 44 & 5 & 0 & 3 & 1 & 0 \\
\hline Jasim Alabbad & 2018 & Kuwait & 148 & - & - & - & 78 & 18 & 26 & 9 & 1 & 8 & 3 \\
\hline Hongna Ren & 2018 & China & 109 & - & - & $4-73$ & 43 & 18 & 11 & 8 & 0 & 13 & 0 \\
\hline Xingwei Sun & 2018 & China & 124 & 114 & 31 & $15-65$ & 90 & 16 & 3 & 4 & 0 & 0 & 1 \\
\hline Jinhui Gu & 2017 & China & 156 & 130 & 16 & $12-72$ & 110 & 18 & 12 & 2 & 0 & 3 & 0 \\
\hline Xuecheng Zhang & 2016 & China & 322 & - & - & - & 137 & 42 & 49 & 13 & 27 & 26 & 0 \\
\hline Saijun Wang & 2014 & China & 91 & 203 & 39 & $15-64$ & 77 & 11 & 0 & 0 & 0 & 0 & 0 \\
\hline Chaolan Luo & 2013 & China & 70 & 60 & 16 & $17-68$ & 54 & 18 & 7 & 10 & 0 & 4 & 0 \\
\hline Yenv Shou & 2013 & China & 176 & 136 & 72 & - & 128 & 23 & 3 & 0 & 0 & 0 & 2 \\
\hline
\end{tabular}

\subsubsection{Statistical analysis}

Meta-analysis was performed by STATA 12.0 statistical software. The effect variable was the detection rate of the three pathogens. The combined statistics of the rates and the $95 \%$ confidence interval (CI) were calculated. The heterogeneity of the literatures was evaluated by $\mathrm{I}^{2}$ test. $p \geq 0.1$ indicates heterogeneity among the literatures, and the fixed effect model would be used. However, $p<0.1$ indicates heterogeneity in each study, and the random effect model would be used. Publication bias was evaluated by the symmetry of the funnel plot.

\section{Results}

\subsection{Literature search results}

A total of 221 domestic and foreign literatures were retrieved in this research, including 114 literatures in the Chinese language and 107 in the English language. Through reading the full text, the article s were 
screened according to the inclusion and exclusion criteria. In regard to the publication time, 89 papers, 29 conference abstracts, 71 papers with less than 70 samples, and 21 papers with unclear bacterial classification were excluded. A total of 11 literatures met the inclusion and exclusion criteria ${ }^{[2-12]}$ and were included in this meta-analysis as shown in Figure 1.

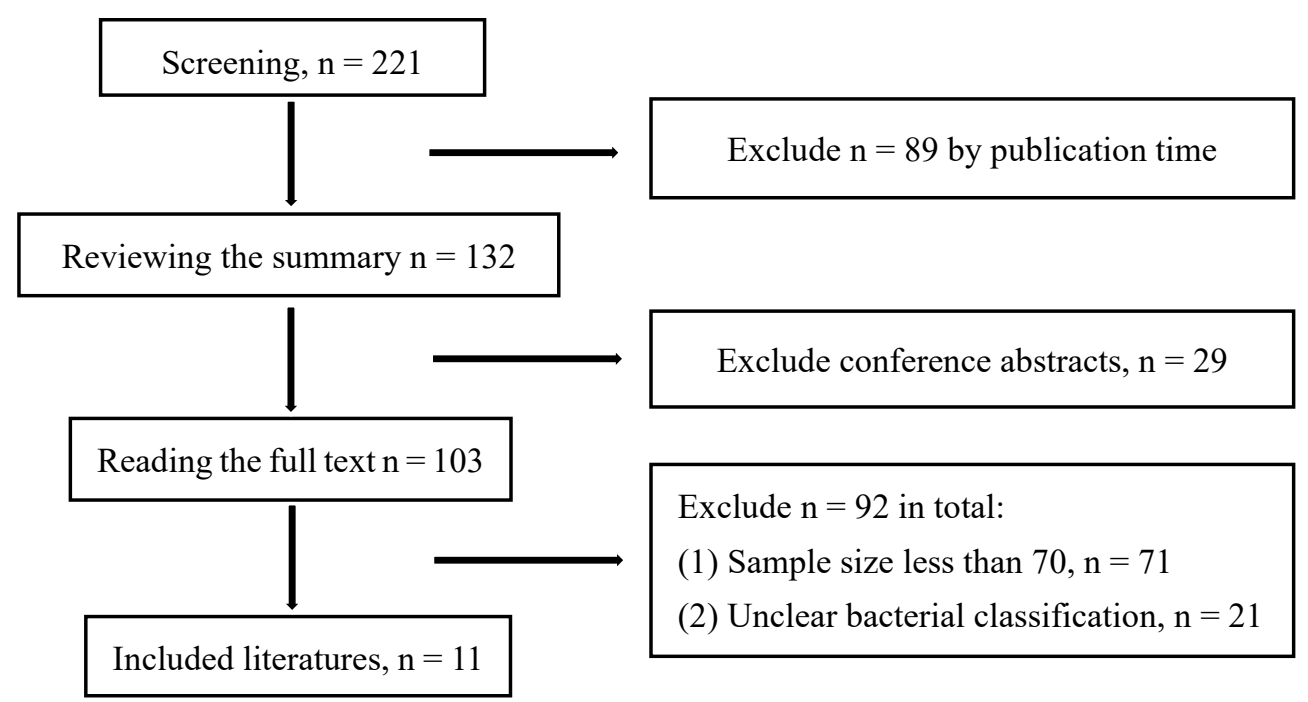

Figure 1. Literature search process

\subsection{Statistical analysis results}

\subsubsection{Bacterial detection rates}

The detection rates and heterogeneity evaluation of combined Escherichia coli, Klebsiella pneumoniae, and Staphylococcus were 65\% (95\% CI, 0.56-0.74), 14\% (95\% CI, 0.12-0.16), and 7\% (95\% CI, 0.04-0.10), respectively as shown in Figure 2, Figure 3, and Figure 4.

\subsubsection{Heterogeneity evaluation}

As shown in Figure 2 and Figure 4, there is heterogeneity in the detection rates of Escherichia coli and Staphylococcus, indicating that there are differences among the studies. The random effect model was adopted. The heterogeneity of Klebsiella pneumoniae is shown in Figure 3, where the heterogeneity among the studies is significantly lesser compared to the first two; thus, the fixed effect model was adopted. 


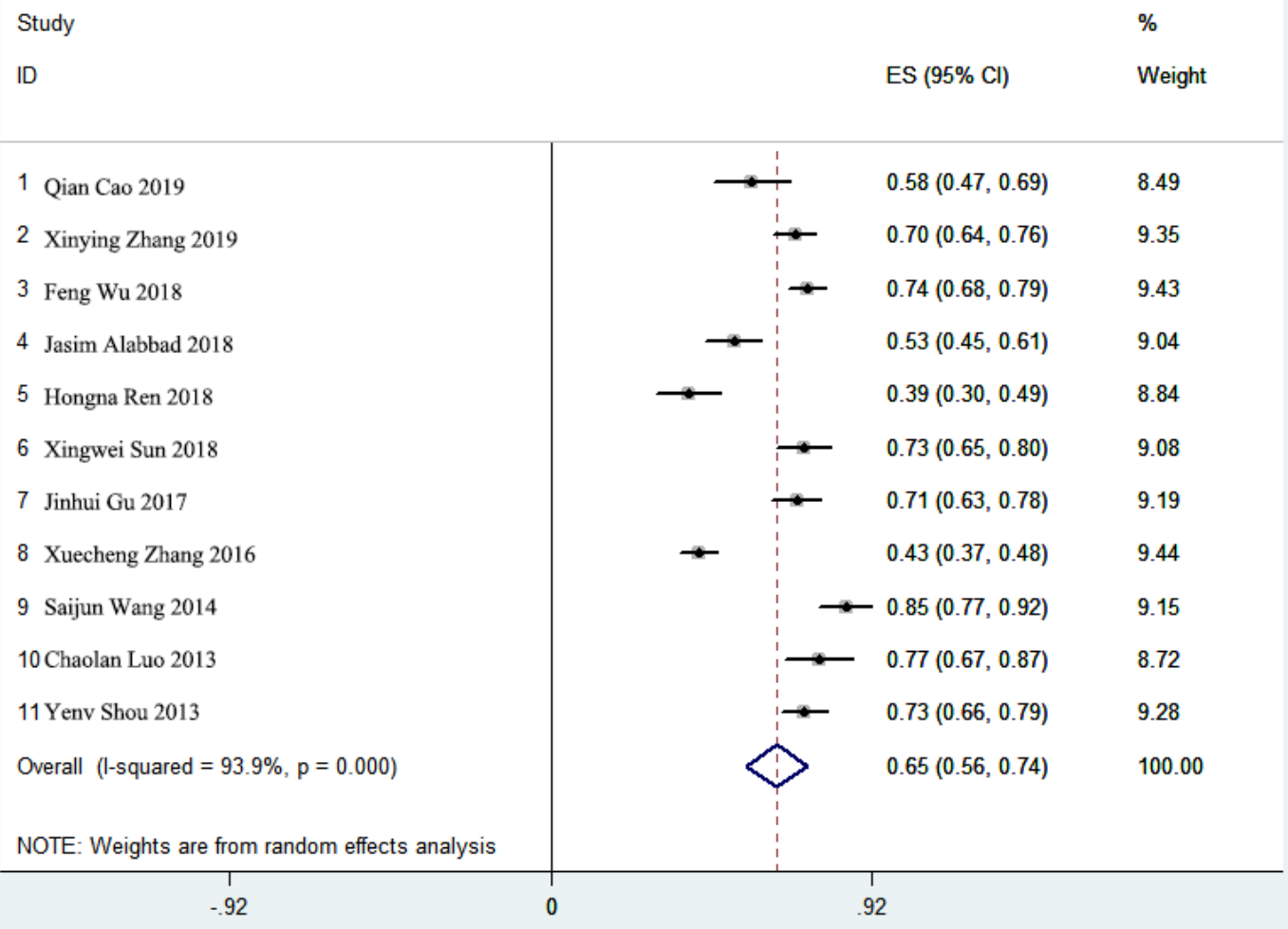

Figure 2. Meta-analysis of the detection rate of Escherichia coli

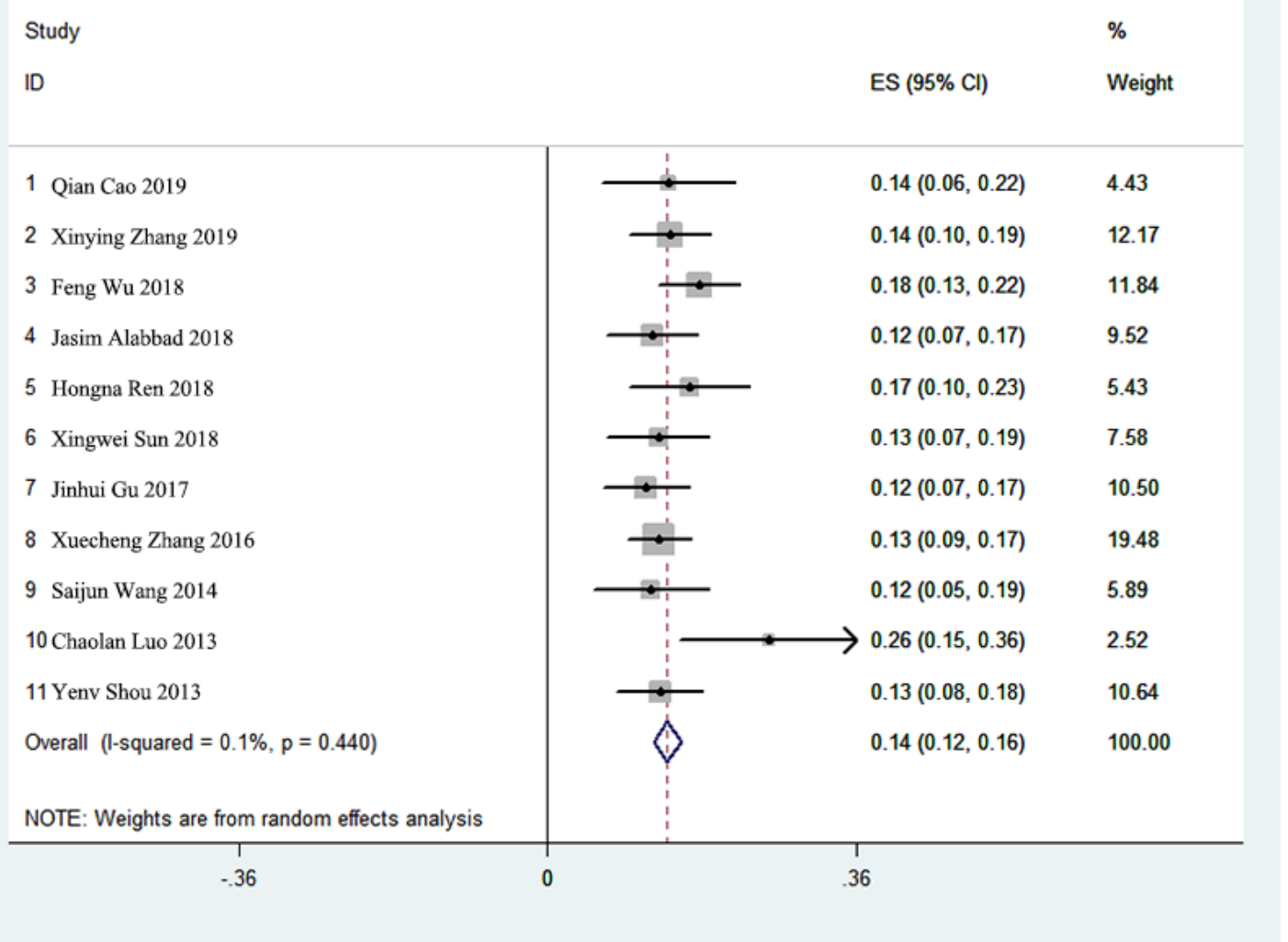

Figure 3. Meta-analysis of the detection rate of Klebsiella pneumoniae 


\begin{tabular}{|c|c|c|c|}
\hline \multicolumn{2}{|l|}{ Study } & & $\%$ \\
\hline ID & & $\mathrm{ES}(95 \% \mathrm{Cl})$ & Weight \\
\hline 1 Qian Cao 2019 & & $0.10(0.04,0.17)$ & 7.68 \\
\hline 2 Xinying Zhang 2019 & $\rightarrow$ & $0.02(0.00,0.04)$ & 11.95 \\
\hline 3 Feng Wu 2018 & $\rightarrow$ & $0.02(0.00,0.04)$ & 12.11 \\
\hline 4 Jasim Alabbad 2018 & & $0.18(0.11,0.24)$ & 8.23 \\
\hline 5 Hongna Ren 2018 & & $0.10(0.04,0.16)$ & 8.68 \\
\hline 6 Xingwei Sun 2018 & & $0.02(-0.00,0.05)$ & 11.44 \\
\hline 7 Jinhui Gu 2017 & & $0.08(0.04,0.12)$ & 10.12 \\
\hline 8 Xuecheng Zhang 2016 & & $0.15(0.11,0.19)$ & 10.36 \\
\hline 10 Chaolan Luo 2013 & & $0.10(0.03,0.17)$ & 7.42 \\
\hline 11 Yenv Shou & 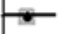 & $0.02(-0.00,0.04)$ & 12.01 \\
\hline 9 Saijun Wang 2014 & & (Excluded) & 0.00 \\
\hline Overall (l-squared $=88.5 \%, p=0.000)$ & & $0.07(0.04,0.10)$ & 100.00 \\
\hline NOTE: Weights are from random effects analysis & & & \\
\hline $\begin{array}{c}1 \\
-.237\end{array}$ & 0 & & \\
\hline
\end{tabular}

Figure 4. Meta-analysis of the detection rate of Staphylococcus

\subsubsection{Publication bias}

The publication bias is shown in Figure 5, Figure 6, and Figure 7, where the funnel plots show a certain publication bias.

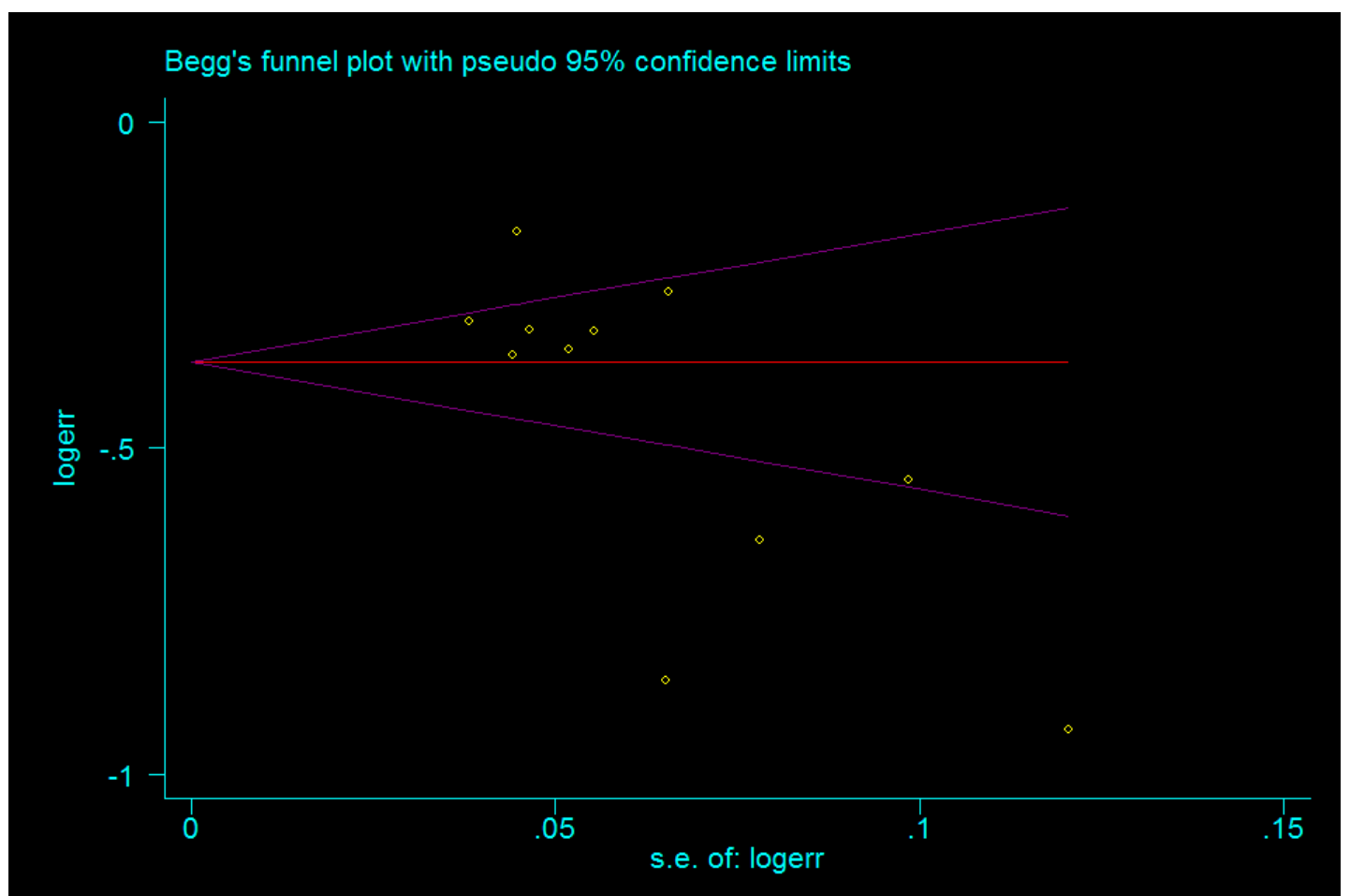

Figure 5. Funnel plot of the publication bias of Escherichia coli detection rate in each study 


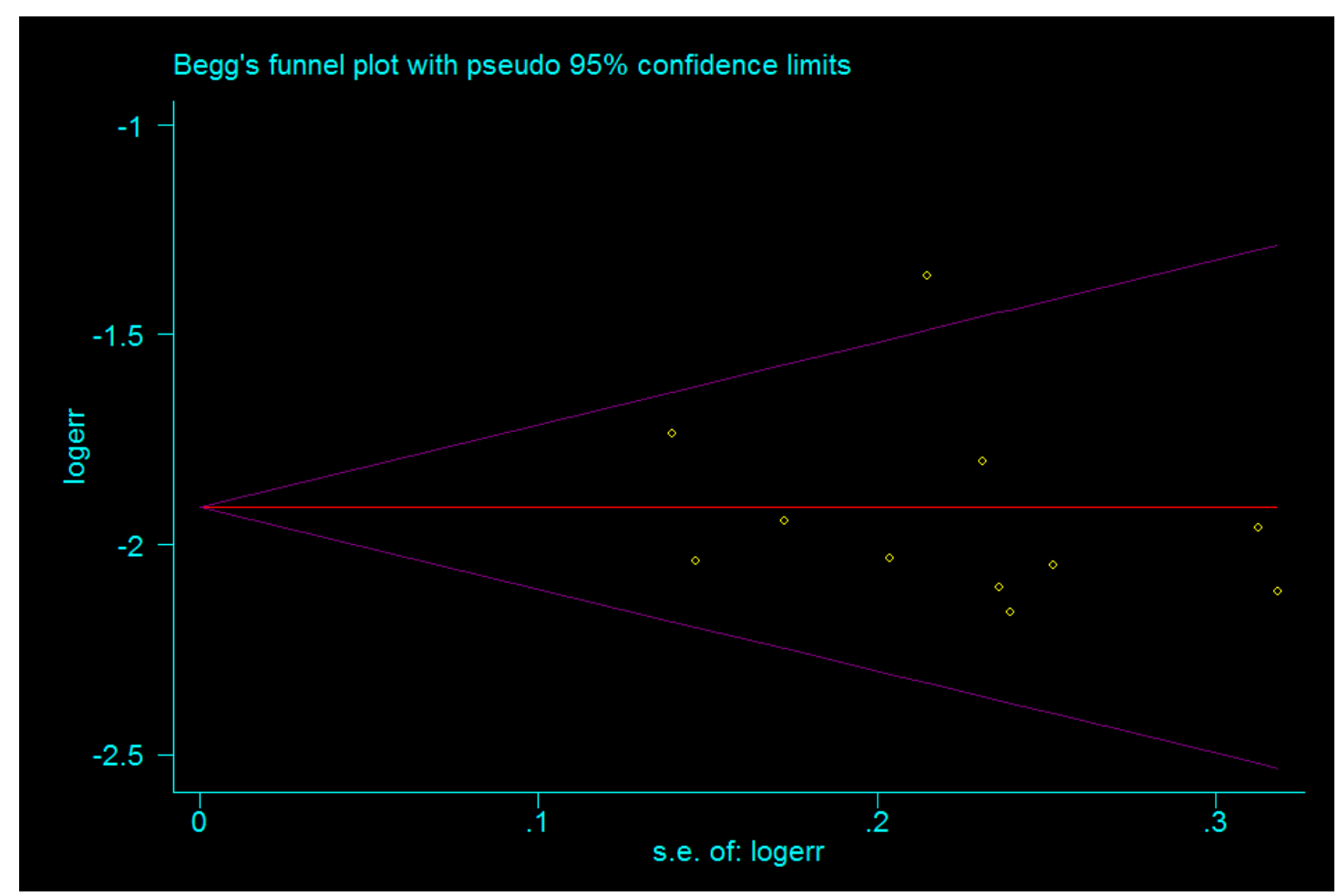

Figure 6. Funnel plot of the publication bias of Klebsiella pneumoniae detection rate in each study

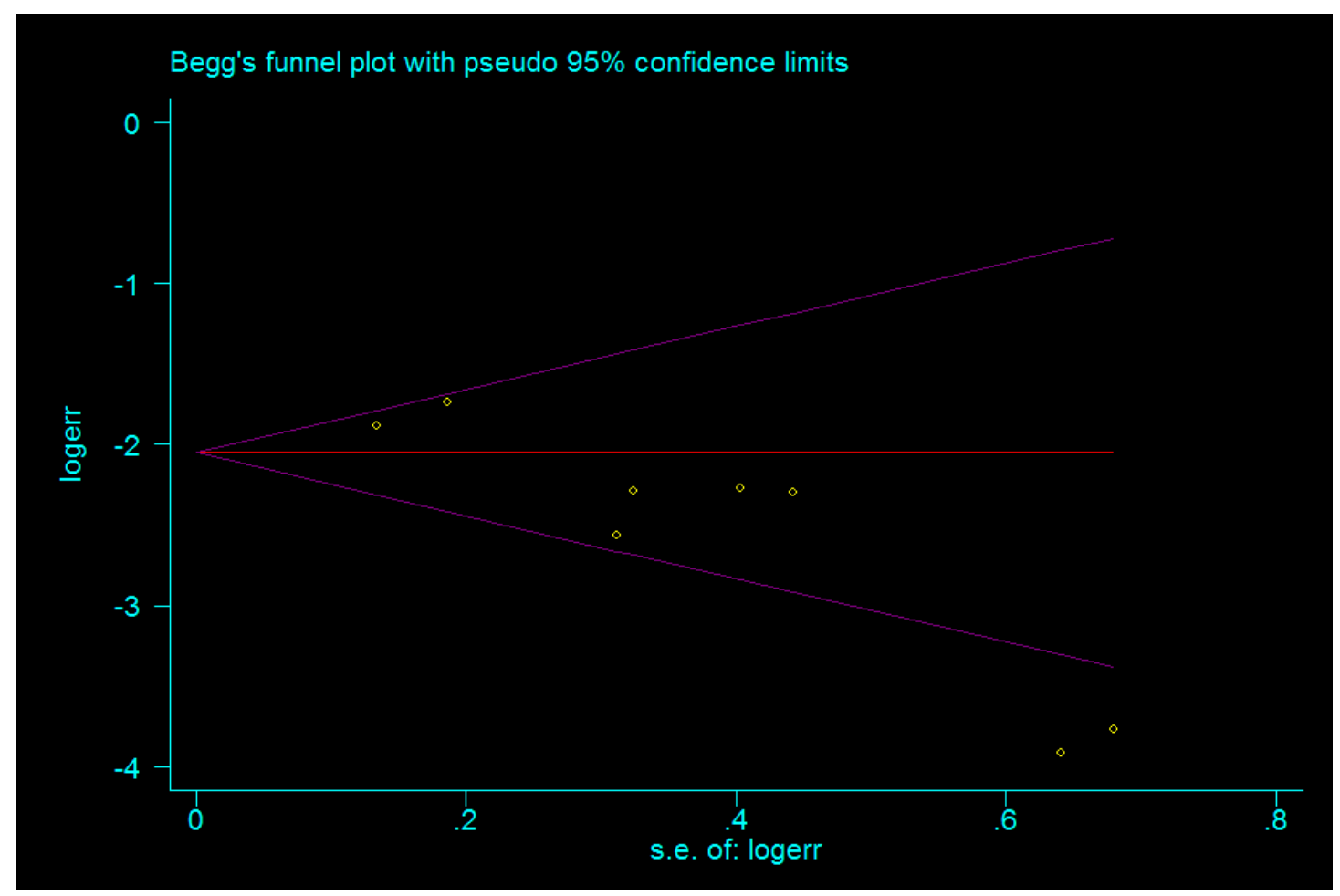

Figure 7. Funnel plot of the publication bias of Staphylococcus detection rate in each study

\section{Discussion}

In this study, the detection rates of the three main pathogens (Escherichia coli, Klebsiella pneumoniae, and Staphylococcus) in 11 literatures ${ }^{[2-12]}$ at home and abroad about the distribution of bacteria in perianal abscess were combined by meta-analysis. The results showed that Escherichia coli, Klebsiella pneumoniae, and Staphylococcus were the main bacteria in the bacterial distribution of perianal abscess, and the 
detection rate of Escherichia coli was the highest, 0.65, indicating that Escherichia coli is the main pathogenic bacteria in the disease process of perianal abscess.

It was also found that many patients preferred the surgical method of incision and drainage (I\&D) due to the inconveniences from work and life. There were no clear guidelines for the use of antibiotics after incision and drainage. They use of antibiotics was only for special cases, such as extensive cellulitis ${ }^{[13]}$. There were many debates about the etiology and prevention of recurrent perianal abscess, especially the role of antibiotics in the development of anal fistula after incision and drainage. Leila Ghahramani and other researchers evaluated the role of postoperative antibiotics in the prevention of anal fistula after I\&D of perianal abscess in their study; there was a significant reduction in the rate of fistula formation among the patients that received preventive antibiotics ${ }^{[14]}$. Meanwhile, in a study carried out by Valentin Mocanu and other researchers, it was found that antibiotic treatment after incision and drainage of anorectal abscess can reduce the probability of fistula formation by 36\%, and the use of antibiotics for 10 days after postoperative drainage can avoid the incidence of fistula formation in other healthy patients ${ }^{[15]}$. However, unnecessary antibiotics can also cause allergic reactions and side effects in patients ${ }^{[16]}$. Although this controversy exists, local and foreign literatures have suggested that bacterial analysis of perianal abscess has certain enlightenment and plays a guiding role in the use of antibiotics after perianal abscess drainage and in patients who do not meet surgical indications. Therefore, the bacterial analysis of perianal abscess has a certain research significance in clinical practice and experiments, but it requires scientific and standardized collection as well as correct statistical analysis.

Due to the majority of Chinese literatures included, the representativeness of the literature research and data analysis has regional limitations. In addition, the heterogeneity of various studies is large. The main reason is that there are obvious differences in the research subjects, research time, and sample size among the studies. The bacterial distribution obtained in this study can provide a certain basis for antibiotic treatment in clinical practice. The specific mode and dose selection require further clinical exploration and experimental research.

\section{Disclosure statement}

The authors declare that there is no conflict of interest.

\section{References}

[1] Wright WF, 2016, Infectious Diseases Perspective of Anorectal Abscess and Fistula-in-Ano Disease. Am J Med Sci, 351(4): 427-434.

[2] Cao X, You ZX, Wang N, et al., 2019, Analysis on the Distribution of Pathogenic Bacteria and Detection of Multiple Drug Resistant Bacteria in Perianal Abscess. Chinese Journal of Current Advances in General Surgery, 22(07): 566-568.

[3] Zhang XY, Sun LM, Su HB, et al., 2019, Analysis on Bacterial Culture and Drug Resistance of Abscess from 214 Cases of Perianal Abscess. Journal of Hunan University of Chinese Medicine, 39(10): 1271 1274.

[4] Wu F, Xiao MC, Liao YH, 2018, Quantitative Test on Bacterial Culture and Drug Sensitivity of Abscess from Perianal Abscess Patients. Global Traditional Chinese Medicine, 11(10): 37-38.

[5] Alabbad J, Abdul Raheem F, Alkhalifa F, et al., 2019, Retrospective Clinical and Microbiologic Analysis of Patients with Anorectal Abscess. Surg Infect (Larchmt), 20(1): 31-34.

[6] Ren HN, Li J, 2018, Distribution and Drug Resistance of Pathogens in Perianal Abscess. Journal of Dalian Medical University, 40(6): 525-528. 
[7] Sun XW, Li YY, Su HB, et al., 2018, Analysis on the Distribution and Drug Sensitivity Test Results of 121 Pathogenic Bacteria in Perianal Abscess Patients. Journal of Hunan University of Chinese Medicine, 38(02): 207-210.

[8] Gu JH, Yang BL, Tang LJ, et al., 2017, Analysis on the results of Pus Culture and Drug Sensitivity Test of 146 Cases of Perianal Abscess. Chinese Journal of Bases and Clinics in General Surgery, 24(07): 877-879.

[9] Zhang XC, Zhang LF, Wang SJ, 2016, Distribution and Drug Resistance Analysis of Pathogenic Bacteria in Perianorectal Abscess. Shandong Medical Journal, 56(40): 64-66.

[10] Wang SJ, Yin L, Yang Y, et al., 2014, Distribution and Drug Resistance of Pathogens in Perianal Abscess. Chinese Journal of Health Laboratory Technology, 24(23): 3479-3480.

[11] Luo CL, Yu SW, Xu Z, et al., 2013, Analysis of Bacterial Culture and Drug Sensitivity in Perianal Abscess Patients (Report of 76 Cases). Journal of Colorectal \& Anal Surgery, 19(02): 96-98.

[12] Shou YN, Zhen GD, Chen JJ, et al., 2013, Monitoring and Analysis of Drug Resistance of Escherichia Coli in Pus Samples of 208 Patients with Perianal Abscess. Chinese Journal of Nosocomiology, 23(10): 2501-2503.

[13] Liu CK, Liu CP, Leung CH, et al., 2011, Clinical and Microbiological Analysis of Adult Perianal Abscess. J Microbiol Immunol Infect, 44(3): 204-208.

[14] Ghahramani L, Minaie MR, Arasteh P, et al., 2017, Antibiotic Therapy for Prevention of Fistula InAno After Incision and Drainage of Simple Perianal Abscess: A Randomized Single Blind Clinical Trial. Surgery, 162(5): 1017-1025.

[15] Mocanu V, Dang JT, Ladak F, et al., 2019, Antibiotic Use in Prevention of Anal Fistulas Following Incision and Drainage of Anorectal Abscesses: A Systematic Review and Meta-Analysis. Am J Surg, 217(5): 910-917.

[16] Seow-En I, Ngu J, 2017, Routine Operative Swab Cultures and Post-Operative Antibiotic Use for Uncomplicated Perianal Abscesses Are Unnecessary. ANZ J Surg, 87(5): 356-359. 